\title{
Comparisons of Computations with Experiments for Electron Transpiration Cooling at High Enthalpies
}

\author{
Kyle M. Hanquist* and Iain D. Boyd ${ }^{\dagger}$ \\ Department of Aerospace Engineering, University of Michigan, Ann Arbor, MI
}

\begin{abstract}
A modeling approach for electron transpiration cooling of high enthalpy flight is compared to a set of experiments performed in a plasma arc tunnel for nitrogen and argon. The comparisons include nitrogen and argon flow at high enthalpies, 12,000 btu/lb and 5,000 btu/lb respectively, with a Mach number of 2.5 to 3. Converting the provided enthalpies and Mach numbers to freestream temperatures and velocities is discussed. The numerical approach is described including implementation of a thermionic emission boundary condition. Also described is the implementation of a finite-rate chemistry model for argon ionization. Different emissive materials are also investigated including graphite and tungsten. The comparisons include two different geometries with different leading edge radii. The numerical results produce a wide range of emitted current due to the uncertainties in freestream conditions and emissive material properties, but still agree well with the experiments. Future work recommendations are provided that may improve the physical accuracy of the modeling capabilities used in the comparisons.
\end{abstract}

\section{Nomenclature}

$A_{R} \quad$ Richardson constant, $1.20 \times 10^{6} \mathrm{~A} / \mathrm{m}^{2} / \mathrm{K}^{2}$

$C_{p} \quad$ Constant pressure specific heat

$C_{s} \quad$ Charge of species $s$

E Electric field

j Electric current density

$J_{e e} \quad$ Emitted electron current density

$\hbar \quad$ Planck constant, $6.63 \times 10^{-34} \mathrm{~m}^{2} \mathrm{~kg} / \mathrm{s}$

$g_{j} \quad$ Degeneracy factor of electronic energy level $j$

$h_{t} \quad$ Total enthalpy

$\Delta h_{i} \quad$ Enthalpy of ionization

$k_{b} \quad$ Backward reaction rate coefficient

$k_{B} \quad$ Boltzmann constant, $1.38 \times 10^{-23} \mathrm{~J} / \mathrm{K}$

$k_{f} \quad$ Forward reaction rate coefficient

$K_{e} \quad$ Equilibrium constant

$m_{s} \quad$ Mass of species $s$

$M \quad$ Mach number

$N_{A v} \quad$ Avogadro constant, $6.02 \times 10^{23} \mathrm{~mol}^{-1}$

$p \quad$ Pressure

$q \quad$ Heat transfer

$Q_{e} \quad$ Elementary charge, $1.60 \times 10^{-19} \mathrm{C}$

$Q_{\text {int }}$ Internal energy partition function

$u \quad$ Freestream velocity

$R_{n} \quad$ Leading edge radius

${ }^{*}$ PhD Candidate, Student Member AIAA.

${ }^{\dagger}$ James E. Knott Professor of Engineering, Fellow AIAA 


$\begin{array}{ll}R_{u} & \text { Universal gas constant, } 8.31 \mathrm{~J} / \mathrm{K} / \mathrm{mol} \\ s & \text { Distance along leading edge } \\ T & \text { Temperature } \\ \dot{w}_{i} & \text { Production rate of species } s \\ \alpha & \text { Level of ionization } \\ \gamma & \text { Ratio of specific heats } \\ \theta_{i} & \text { Characteristic temperature for ionization } \\ \theta_{j} & \text { Characteristic temperature for electronic energy level } j \\ \phi & \text { Electric potential } \\ \Phi & \text { Material work function } \\ \rho & \text { Density } \\ \sigma & \text { Electrical conductivity } \\ \text { Subscript } \\ e & \text { Electron } \\ i & \text { Ion } \\ t r & \text { Translational } \\ v i b & \text { Vibrational } \\ w & \text { Wall }\end{array}$

\section{Introduction}

HYPERSONIC vehicles, such as the Hypersonic Technology Vehicle 2 (HTV-2), require a sharp leading edge

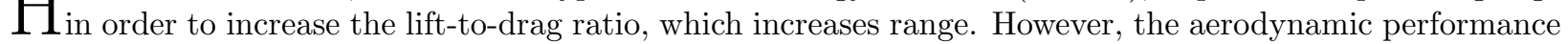
gains offered by sharp leading edges come at the cost of immense, localized heating rates. ${ }^{1}$ One approach to address this issue is to use a leading edge material that can withstand the high heating rates and temperatures. A type of material that meets this criteria is ultra-high temperature composite (UHTC) materials, which were used on the NASA X-43 experimental hypersonic aircraft. ${ }^{2}$ Although UHTC materials have good thermal properties, they have some physical limitations such as heavy weight and weak fracture toughness that suggest they are not the ideal approach. ${ }^{3}$ Another approach to manage the heat loads is to reject the heat passively, either through heat-shield ablation or radiation. Ablation has significant heat management benefits through essentially a controlled melt of the heat shield while also being light-weight, which makes it a great approach for re-entry flight. This shape change of the surface, while permitted for the blunt bodies of re-entry flight, is unacceptable for the sharp leading edges of hypersonic vehicles. Heat management through radiation does not incur shape change, but is limited by the Stefan-Boltzmann law.

An alternative approach that has been recently proposed involves using thermo-electric materials at the leading edges of hypersonic vehicles. When exposed to high convective heating rates, these materials emit a current of electrons that may lead to a transpiration cooling effect of the surface of the vehicle. This phenomenon is known as thermionic emission and occurs when the thermal energy given to the electrons is greater than the binding potential of the surface material. A recent conceptual study was completed and showed thermionic emission can reduce the surface temperature by approximately $40 \%$ for a Mach 19.4 flow over a sharp leading radius at an altitude of $60 \mathrm{~km}$ with a material work function of $2.0 \mathrm{eV}{ }^{4}$ Given the promising trends in that study, further research is needed to improve the modeling capabilities and to validate the numerical approach. Although using thermo-electric materials as a mechanism to reduce the thermal load on hypersonic vehicles is a recent approach, employing thermionic emission in high speed flight is not a novel concept. In the 1960s there was a push to use thermo-electric materials on the nose of re-entry vehicles and collect the emitted electrons as a source of power generation..$^{5,6}$ Experiments were performed using the plasma arc tunnel at Sandia Corporation using a range of different flow conditions, emissive materials, and geometries. ${ }^{7}$ This study aims to assess the computational fluid dynamics (CFD) modeling approach using these experiments.

\section{Numerical Approach}

The numerical simulations in this work are performed using the CFD code LeMANS, which was developed at the University of Michigan. ${ }^{8}$ LeMANS is a parallel, three-dimensional code that solves the laminar Navier- 
Stokes equations on unstructured computational grids. LeMANS includes thermo-chemical non-equilibrium effects and the flow is modeled assuming that the continuum approximation is valid. It is also assumed that the translational and rotational energy modes can be described by a single temperature, $T_{t r}$, and that the vibrational, electronic, and electron translational energy modes are described by a different temperature, $T_{v i b}$. The mixture transport properties are calculated using Wilke's semi-empirical mixing rule ${ }^{9}$, species thermal conductivities determined using Eucken's relation, ${ }^{10}$ and species viscosities determined using Blottner's curve fits. ${ }^{11}$

In LeMANS, the set of governing equations are solved using the finite-volume method applied to unstructured grids with second order spatial accuracy. A modified Steger-Warming Flux Vector Splitting scheme ${ }^{12}$ is used to discretize the inviscid fluxes across cell faces. The viscous terms are computed using cell-centered and nodal values. The viscous stresses are modeled assuming the flow is a Newtonian fluid and Stokes' hypothesis is applicable, and the heat fluxes are modeled according to Fourier's law for all temperatures. For parallel execution of LeMANS, METIS ${ }^{13}$ is used to partition the computational mesh, and the Message Passing Interface (MPI) is used to communicate the necessary information between processors.

A standard finite-rate chemistry model is used for reactive nitrogen $\left(N_{2}, N, N_{2}^{+}, N^{+}\right.$and $\left.e^{-}\right)$, and Park's two-temperature model ${ }^{14}$ is used to account for the effects of thermal nonequilibrium on the forward and backward reactions rates. A finite-rate chemistry model is also implemented into the numerical method to model the electron-impact ionization reactions for argon. The forward reaction rate coefficient is given by, ${ }^{15}$

$$
k_{f}(T)=2.3 \times 10^{34} T^{-3.60} \exp \left(\frac{-182,890}{T}\right)\left[\mathrm{cm}^{3} /(\mathrm{mol} \mathrm{s}]\right.
$$

The backward reaction rate coefficient is then calculated from the equilibrium constant,

$$
K_{e}(T)=\frac{k_{f}(T)}{k_{b}(T)}
$$

The equilibrium constant for the electron-impact ionization reaction of argon is given in Ref. 16. When the chemistry only has an electron-impact ionization reaction, such as with argon, the convergence of the CFD calculation is sensitive to avalanche ionization. ${ }^{14}$ This avalanche process, or chain reaction, occurs when the equation becomes active, the high thermal speed of the electrons result in intrinsically high forward rate coefficients causing the electron density to increase exponentially. This numerical explosion may lead to numerical divergence of the solution. A simple way to negate the probability of numerical explosion is to slowly introduce the electron-impact ionization reaction, which in this study is achieved by ramping the forward reaction rate constant.

\section{A. Electron Emission}

A boundary condition is implemented into LeMANS to model thermionic emission at the material surface. The production rate of electrons is calculated by,

$$
\dot{w}_{e}=\frac{J_{e e}}{Q_{e} N_{A v}}-\sum_{s=i o n s} \dot{w}_{s}
$$

where $J_{e e}$ is the emission current density, $Q_{e}$ is the elementary charge, $N_{A v}$ is the Avogadro constant, and $\dot{w}_{i}$ is the recombination rate of ions. The ions at the surface combine with the emitted electrons to form corresponding neutrals. The current density is calculated using Richardson's Law: ${ }^{17}$

$$
J_{e e}=A_{R} T_{w}^{2} \exp \left(\frac{-\Phi Q_{e}}{k_{B} T_{w}}\right)
$$

where $A_{R}$ is a material-constant assumed to be equal to $1.20 \times 10^{6} \mathrm{~A} / \mathrm{m}^{2} / \mathrm{K}^{2}$ in the current work, $T_{w}$ is the surface temperature, $k_{B}$ is the Boltzmann constant, and $\Phi$ is the material work function defined as the minimum energy required to remove an electron from the material. In the current work, the work function varies between $4.32 \mathrm{eV}$ (lower bound of tungsten) and $5.0 \mathrm{eV}$ (upper bound of graphite). ${ }^{18}$ It is to be noted that in the current work, electrons can only be emitted from the surface using Richardson's law and are assumed not to recombine back to the surface. The recombination rate of ions is calculated assuming that the surface is fully catalytic to ions: ${ }^{19}$ 


$$
\dot{w}_{i}=\frac{\rho_{i}}{m_{i}} \sqrt{\frac{R_{u} T_{w}}{2 \pi m_{i}}}
$$

where $\rho_{i}$ is the ion density, $m_{i}$ is the ion mass, and $R_{u}$ is the universal gas constant. The emitted electrons will carry away energy from the vehicle surface at a flux of:

$$
q_{\text {emiss }}=J_{e e}\left(\Phi+\frac{2 k_{B} T_{w}}{Q_{e}}\right)
$$

\section{B. Electric Field}

The electric field can be replaced by an electric potential, $\phi$, as,

$$
\vec{E}=-\nabla \phi
$$

where the electric potential is calculated using Ohm's law and solving the steady state current continuity equation,

$$
\begin{gathered}
\vec{j}=\sigma \vec{E} \\
\nabla \cdot \vec{j}=\nabla \cdot(\sigma \nabla \phi)=0
\end{gathered}
$$

where $\sigma$ is the electrical conductivity of the plasma. The electrical conductivity in this work is approximated using a semi-empirical model developed by Razier ${ }^{20}$ which is valid for air, nitrogen, and argon,

$$
\sigma=8300 \times \exp \left(\frac{-36,000}{T}\right)
$$

A zero-gradient condition is used at the inlet, outlet, and symmetry computational boundaries for the electric potential. At the wall, the electric potential is calculated by assuming the flowfield ion and electron fluxes are equal at the wall, ${ }^{21,22}$

$$
\phi_{w}=\frac{k_{B} T_{e}}{Q_{e}} \log \left(\frac{n_{i}}{n_{e}} \sqrt{\frac{2 \pi m_{e}\left(T_{i}+T_{e}\right)}{m_{i} T_{e}}}\right)
$$

where $n_{i}$ and $n_{e}$ are the ion and electron number densities, $T_{i}$ and $T_{e}$ are ion and electron temperatures (assumed to be equal to $T_{w}$ in this work), and $m_{i}$ and $m_{e}$ are the ion and electron masses. The effect of thermionic emission is not included in this calculation, which will have an influence on the wall potential.

\section{Test Case Description}

The experiments of Touryan were performed in Sandia Corporation's plasma arc tunnels in the 1960s. A detailed description of the experiments are given in Refs. 5 and 7 . The experiments investigate the effect of different freestream conditions, emissive materials, and geometries on thermionic emission.

\section{A. Freestream Conditions}

The experiments examined the effect of different enthalpies and working fluids on thermionic emission. The freestream properties cited in the Touryan experiments are given in Table 1.

Table 1: Freestream properties of Touryan experiments ${ }^{5}$

\begin{tabular}{cccc}
\hline Fluid & Enthalpy, btu/lb & Mach Number & Pressure, atm \\
\hline Nitrogen & 12,000 & $2.5-3$ & 0.01 \\
Argon & 5,000 & & \\
\hline
\end{tabular}


The numerical method employed requires that the freestream properties are described in terms of temperature, velocity, and density. The following section explains how these properties are converted from Mach number, pressure, and total enthalpy for both nitrogen and argon.

\section{Nitrogen}

In order to convert the freestream properties for nitrogen, NASA's Chemical Equilibrium with Applications (CEA) computer program is utilized. ${ }^{23}$ This approach accounts for both dissociation and ionization of nitrogen. This approach also assumes the freestream flow is in equilibrium, which is not necessarily the case. The converted freestream properties are given in Table 2. The three different Mach numbers are used to cover the range of uncertainty in Mach number, with each Mach number giving different freestream conditions. Electrons are also included in the freestream to ensure the freestream is charge neutral.

Table 2: Converted freestream properties for nitrogen

\begin{tabular}{ccccc|cccc}
\hline $\begin{array}{c}\text { Enthalpy, } \\
\text { btu/lb }\end{array}$ & $\begin{array}{c}\text { Mach } \\
\text { Number }\end{array}$ & $\begin{array}{c}\text { Temperature, } \\
\mathrm{K}\end{array}$ & $\begin{array}{c}\text { Velocity, } \\
\mathrm{km} / \mathrm{s}\end{array}$ & $\begin{array}{c}\text { Density, } \\
\mathrm{kg} / \mathrm{m}^{3}\end{array}$ & \multicolumn{4}{|c}{ Mass fraction } \\
\hline \multirow{3}{*}{12,000} & 2.5 & 5430 & 3.93 & $4.53 \times 10^{-4}$ & $3.87 \times 10^{-1}$ & $5.01 \times 10^{-5}$ & $6.12 \times 10^{-1}$ & $1.05 \times 10^{-5}$ \\
& 2.75 & 5380 & 4.25 & $4.69 \times 10^{-4}$ & $3.53 \times 10^{-1}$ & $3.97 \times 10^{-5}$ & $6.47 \times 10^{-1}$ & $9.38 \times 10^{-6}$ \\
& 3 & 5320 & 4.55 & $4.87 \times 10^{-4}$ & $3.18 \times 10^{-1}$ & $3.08 \times 10^{-5}$ & $6.82 \times 10^{-1}$ & $8.26 \times 10^{-6}$ \\
\hline
\end{tabular}

\section{Argon}

A different approach is used to convert the freestream properties for argon. NASA's Chemical Equilibrium with Applications computer program currently does not have the capability to account for ionization of argon. Treating argon as an ideal gas, the enthalpy and Mach number can be converted to a temperature and velocity using the following relations:

$$
\begin{gathered}
h_{t}=C_{p, A r} T+\frac{M^{2} \gamma R_{A r} T}{2} \\
u=M \sqrt{\gamma R_{A r} T} \\
p=\rho R_{A r} T
\end{gathered}
$$

where $h_{t}$ is the total enthalpy, $C_{p, A r}$ is the constant pressure specific heat of argon, $M$ is the Mach number, $u$ is the freestream velocity, $\gamma$ is the ratio of specific heats, and $p$ is the freestream pressure. The resulting freestream properties without ionization are given in Table 3.

Table 3: Converted freestream properties for argon without ionization

\begin{tabular}{ccccc}
\hline $\begin{array}{c}\text { Enthalpy, } \\
\text { btu/lb }\end{array}$ & $\begin{array}{c}\text { Mach } \\
\text { Number }\end{array}$ & $\begin{array}{c}\text { Temperature, } \\
\mathrm{K}\end{array}$ & $\begin{array}{c}\text { Velocity, } \\
\mathrm{km} / \mathrm{s}\end{array}$ & $\begin{array}{c}\text { Density, } \\
\mathrm{kg} / \mathrm{m}^{3}\end{array}$ \\
\hline \multirow{3}{*}{5,000} & 2.5 & 7250 & 3.96 & $6.69 \times 10^{-4}$ \\
& 2.75 & 6350 & 4.08 & $7.64 \times 10^{-4}$ \\
& 3 & 5590 & 4.18 & $8.68 \times 10^{-4}$ \\
\hline
\end{tabular}

The equilibrium level of ionization for argon can be calculated using Saha's equation, ${ }^{10}$

$$
\frac{\alpha^{2}}{1-\alpha^{2}}=\frac{1}{p}\left(\frac{2 \pi m_{e}}{\hbar^{2}}\right)^{3 / 2}\left(k_{B} T\right)^{5 / 2} \frac{2 Q_{i n t}^{A r^{+}}}{Q_{\text {int }}^{A r}} \exp \left(\frac{-\theta_{i}}{T}\right)
$$




$$
Q_{i n t} \approx g_{0}+g_{1} \exp \left(\frac{-\theta_{1}}{T}\right)+g_{2} \exp \left(\frac{-\theta_{2}}{T}\right)
$$

where $Q_{i n t}$ is the internal partition function, which is equal to the electronic partition function in the case of argon, $g_{j}$ are the degeneracy factors and $\theta_{j}$ are the characteristic temperatures for electronic excitation of energy level $j$, and $\theta_{i}$ is the ionization energy. Table 4 lists the constants used to calculate the electronic partition function and equilibrium level of ionization. ${ }^{24}$

Equation 12 can be modified to account for enthalpy of ionization assuming that the specific heat of argon ions and neutrals are equal,

$$
h_{t}=C_{p, A r} T+\frac{M^{2} \gamma R T}{2}+\alpha \Delta h_{i}
$$

where $\Delta h_{i}$ is the enthalpy of ionization for argon.

Table 4: Constants used to calculate equilibrium level of ionization for argon

\begin{tabular}{c|ccc}
\hline & & $A r$ & $A r^{+}$ \\
\hline$\theta_{i}, K$ & 183,000 & & \\
$\theta_{1}, K$ & & 134,061 & 2,061 \\
$\theta_{2}, K$ & & 134,934 & 156,478 \\
\hline$g_{0}$ & & 1 & 4 \\
$g_{1}$ & 5 & 2 \\
$g_{2}$ & 3 & 2 \\
\hline
\end{tabular}

The resulting freestream properties accounting for ionization are given in Table 5. Accounting for ionization results in slightly lower freestream temperatures and velocities, especially for the Mach 2.5 case. This approach also assumes that the flow is in equilibrium, which is not necessarily the case. Although the level of ionization is low, it is still useful to include charged particles in the freestream for stability of the numerical method and also for the recombination of the ions to the assumed fully-catalytic material surface.

Table 5: Converted freestream properties for argon with ionization

\begin{tabular}{cccccc}
\hline $\begin{array}{c}\text { Enthalpy, } \\
\text { btu/lb }\end{array}$ & $\begin{array}{c}\text { Mach } \\
\text { Number }\end{array}$ & $\begin{array}{c}\text { Temperature, } \\
\mathrm{K}\end{array}$ & $\begin{array}{c}\text { Velocity, } \\
\mathrm{km} / \mathrm{s}\end{array}$ & $\begin{array}{c}\text { Density, } \\
\mathrm{kg} / \mathrm{m}^{3}\end{array}$ & $\begin{array}{c}\text { Level of } \\
\text { Ionization }\end{array}$ \\
\hline \multirow{3}{*}{5,000} & 2.5 & 7170 & 3.94 & $6.80 \times 10^{-4}$ & $3.59 \times 10^{-3}$ \\
& 2.75 & 6340 & 4.08 & $7.68 \times 10^{-4}$ & $5.78 \times 10^{-4}$ \\
& 3 & 5590 & 4.18 & $8.71 \times 10^{-4}$ & $7.08 \times 10^{-5}$ \\
\hline
\end{tabular}

\section{B. Emissive Material}

The experiments investigated the effect of using different emissive materials on thermionic emission. The two materials used and their corresponding work functions (energy required to remove an electron) are given in Table 6 . The work functions are significantly higher than those in the previous computational study (2-2.4 eV). ${ }^{4}$ 
Table 6: Emissive material properties

\begin{tabular}{|c|c|c|c|}
\hline Mate & Work funct & $\mathrm{n}, \mathrm{eV}$ & Refs. \\
\hline Gra & 4.65 & 5.0 & $25,26,18$ \\
\hline Tun & 4.32 & 4.65 & 18,27 \\
\hline
\end{tabular}

\section{Geometry}

The experiments used two different geometries, denoted S-6 and S-30. The S-30 geometry has a sharper nose radius and a larger emitter area. The S-6 geometry is an axisymetric cone with a $0.73 \mathrm{~cm}$ leading nose radius, followed by a 10 degree angle wedge, a cylinder region, and a 6 degree angle wedge as shown in Fig. 1. The geometry is split into two regions, the emitter and collector region. The emitter region usually consists of a material with a lower work function than the collector region. The emitter region surface area is 1.3 in $^{2}$ consisting of the leading nose radius and the 10 degree angle wedge. For this work, the emitter and collector region are the same material, graphite. The S-30 geometry is an axisymmetric cone with a $1.0 \mathrm{~mm}$ leading nose radius, followed by a 13.5 degree angle wedge as shown in Fig. 2. The whole geometry is considered the emitted region and has a surface area of $2.48 \mathrm{in}^{2}$. The material used for this geometry is tungsten.

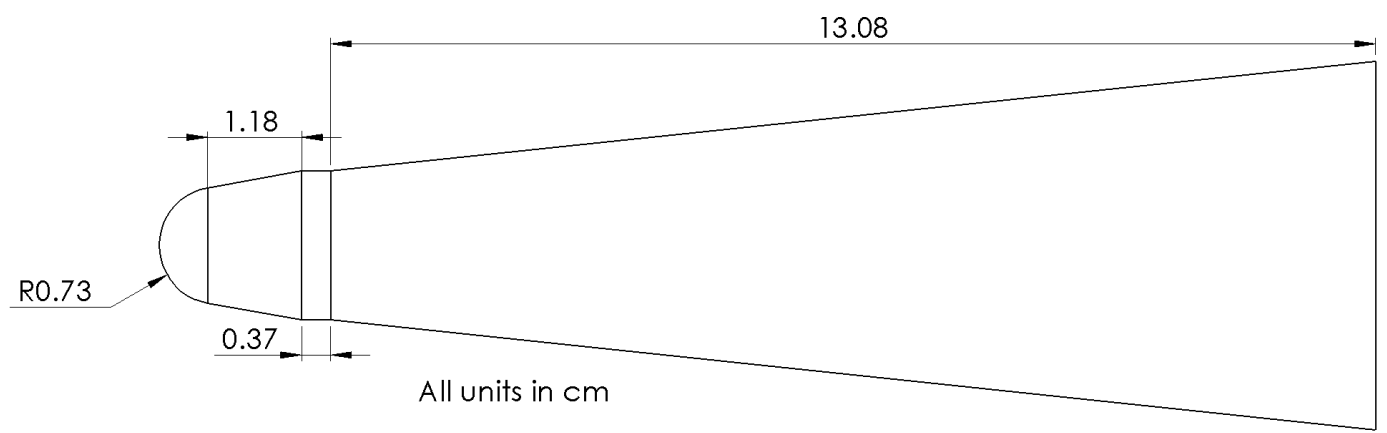

Figure 1: S-6 geometry

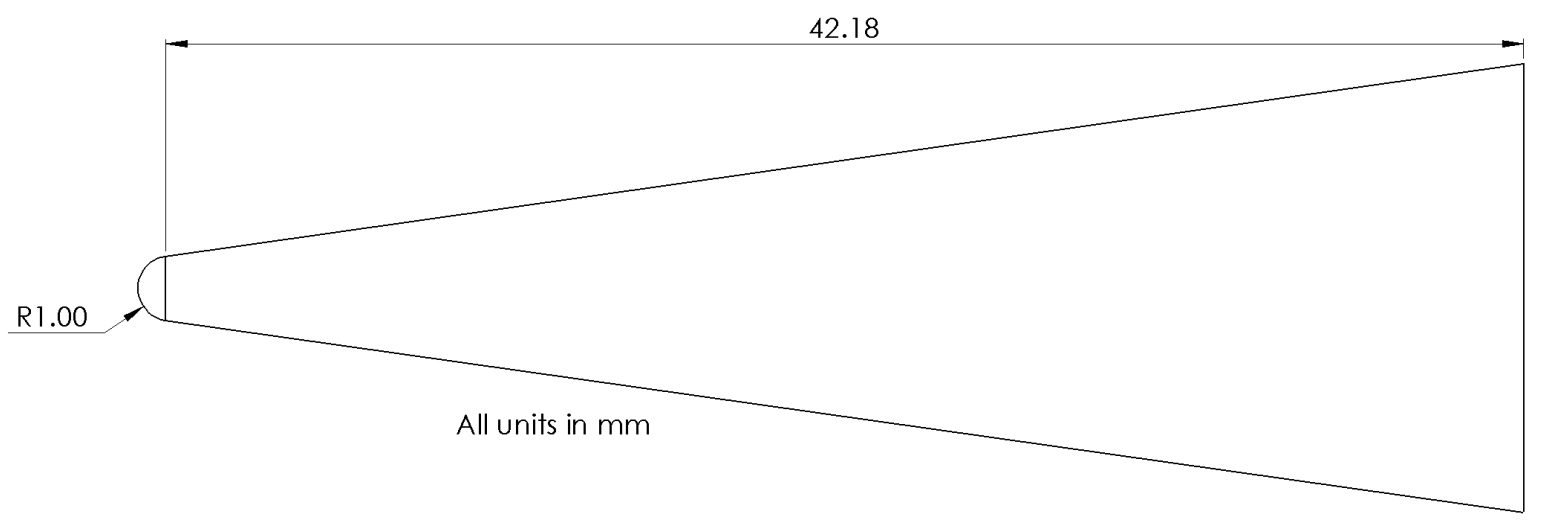

Figure 2: S-30 geometry

Grids are generated for both geometries, and a grid convergence study revealed that the solution is grid-independent using these meshes. The computational grid used for the S- 6 geometry is axisymmetric and composed of approximately 21,000 cells, with 130 cells in the axial direction and 160 cells in the radial direction. The computational grid used for the S-30 geometry is also axisymmetric and composed of approximately 28,000 cells, with 154 cells in the axial direction and 180 in the radial direction. 


\section{Numerical Results}

The goal of this study is to compare current thermionic electron emission modeling approaches to previous experiments using a range of different freestream conditions, emissive materials, and geometries.

The flowfield features for the conditions investigated in this study are shown in Figs. 3 and 4 . Figure 3 shows the temperature contours for the Mach 2.75 nitrogen cases for both the S-6 and S-30 geometry with and without emission. The top half corresponds to the results obtained without thermionic emission, and the bottom half is for the results obtained with thermionic emission. The case without emission is analogous to using a thermo-electric material with an infinite work function or a work function that is much greater than the surface temperature. The material for the S-6 geometry is graphite and the material for the S-30 geometry is tungsten. For the nitrogen cases, the fluid temperature rises to above $12,000 \mathrm{~K}$ for the S-6 geometry and above 10,000 K for the S-30 geometry across the bow shock before decreasing in the shock layer. The S-6 geometry bow shock temperature is expected to be higher due to the more blunt leading edge, as seen. Figure 4 shows the temperature contours for the Mach 2.75 argon cases for both the S-6 and S-30 geometries with and without emission. The fluid temperature is much hotter for argon with 20,000 K for the S-6 geometry and above 18,000 K for the S-30 geometry across the bow shock before decreasing in the shock layer. This is also expected due to the much higher freestream temperature of argon. The nitrogen cases also can dissipate energy through dissociation, which in effect lowers the temperature.

The temperature and heat transfer distributions along the vehicle surface are presented in Figs. 5 and 6 . Note that the distance along the leading edge is normalized by the leading edge radius. Figure 5 shows the surface temperature and convective heat transfer for the Mach 2.75 nitrogen cases. For the S-6 geometry, emission with a work function corresponding to $4.8 \mathrm{eV}$ results in an increase in surface temperature and a decrease in surface heat transfer. It is expected that a higher surface temperature corresponds to a smaller convective heat transfer because a smaller temperature gradient will result in less heat transfer due to Fourier's Law. In previous electron transpiration cooling analysis, however, electron emission resulted in lower surface temperatures with higher convective heat transfer. ${ }^{4}$ This trend is seen for the nitrogen S-30 geometry, where emission with a work function corresponding to $4.48 \mathrm{eV}$ results in a decrease in surface temperature and an increase in convective heat transfer. This suggests that electron emission does not always lower the surface temperature but depends on the amount of emission given by the material work function and flow conditions. This is similar to the Nottingham Effect primarily seen in field emission, where the energy balance resulting from the difference between the average energy of the emitted electrons and that of the replacement electrons results in either surface heating or cooling. ${ }^{28}$ The Mach 2.75 argon cases surface temperatures and convective heat transfer are shown in Fig. 6. For the S-30 geometry, emission with a work function of $4.48 \mathrm{eV}$ results in a lower surface temperature and higher convective heat transfer, which is the same trend as the nitrogen case for this geometry. For the S-6 geometry, emission with a work function of $4.8 \mathrm{eV}$ also results in a slightly lower surface temperature and higher heat transfer, which is the opposite trend of the nitrogen case. The argon cases have higher surface temperatures than the nitrogen cases, which results in an higher level of emission as shown in Fig. 7. The S-30 cases have significantly more emission which is due the sharper leading edge and also the lower work function of tungsten. 


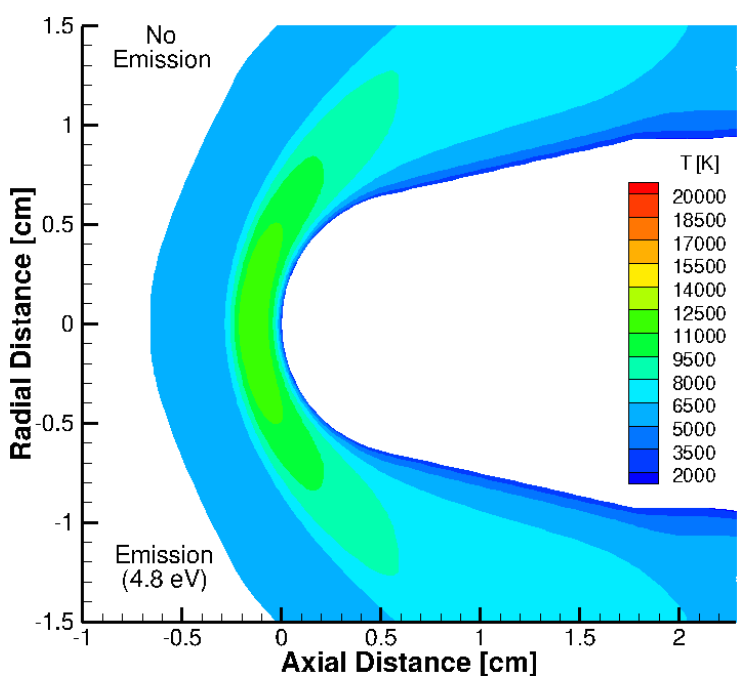

(a) S-6

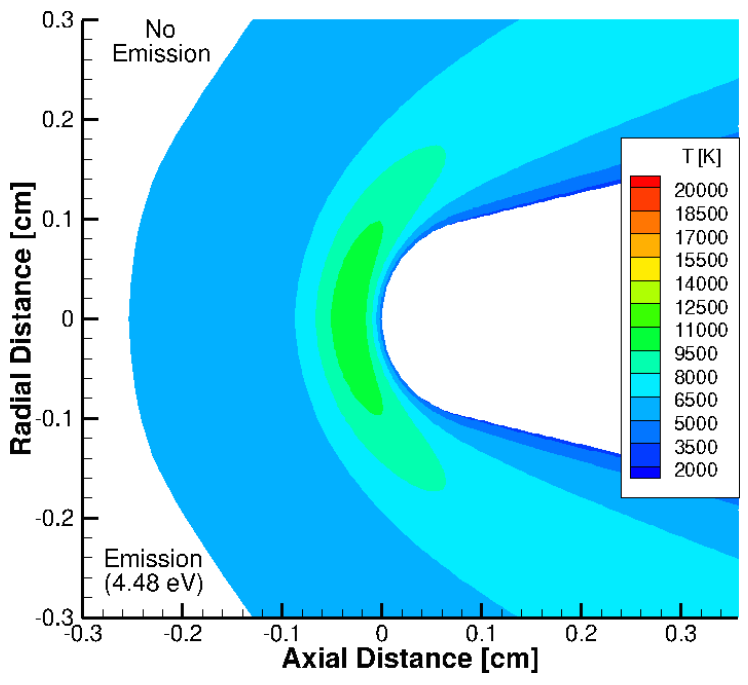

(b) S-30

Figure 3: Temperature contours for Mach 2.75 nitrogen: without emission (top), with emission (bottom).

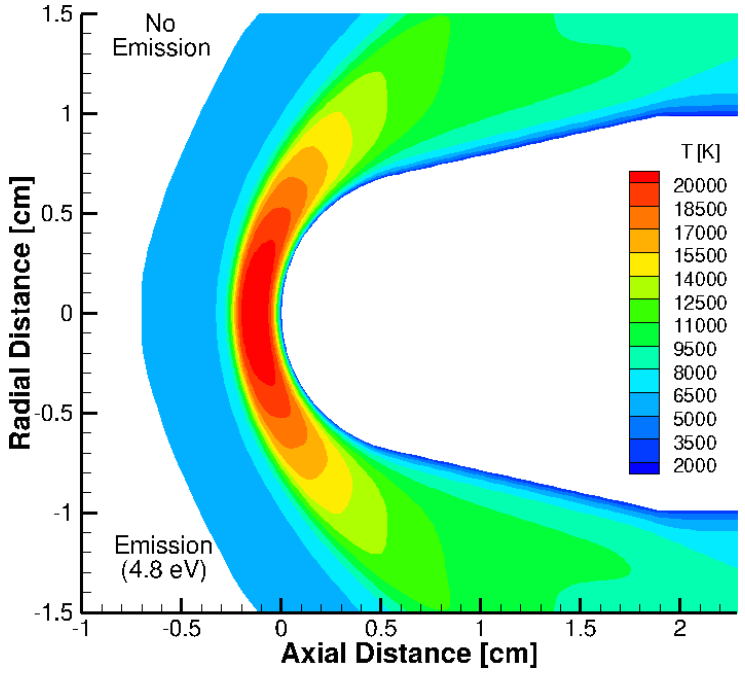

(a) S-6

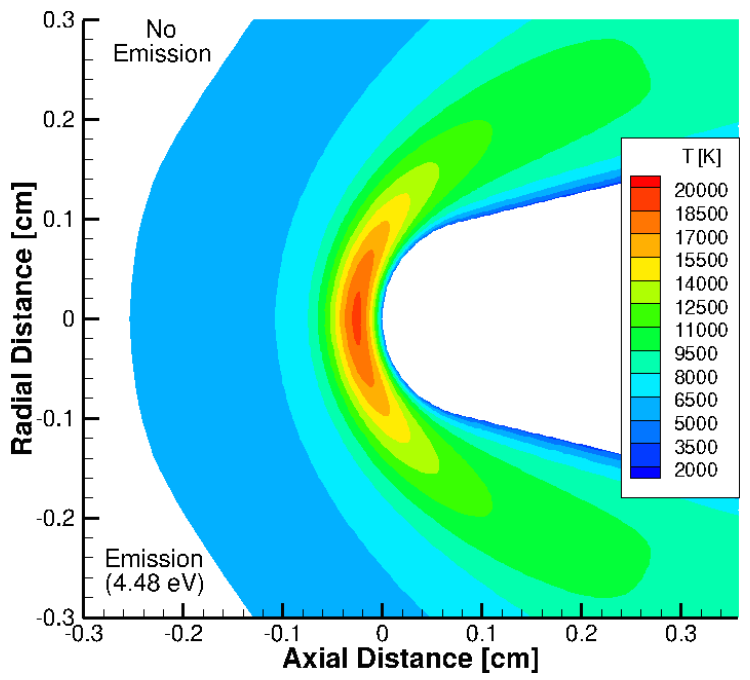

(b) S-30

Figure 4: Temperature contours for Mach 2.75 argon: without emission (top); with emission (bottom). 


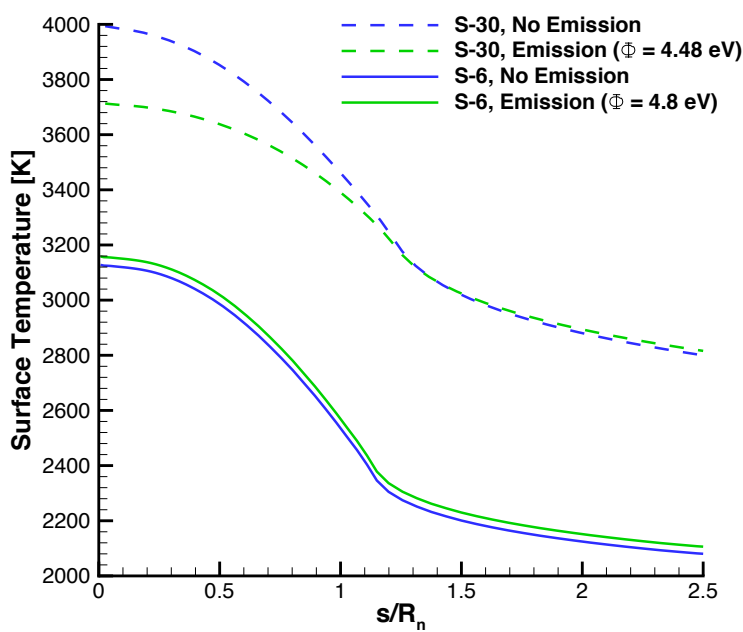

(a) Surface temperature

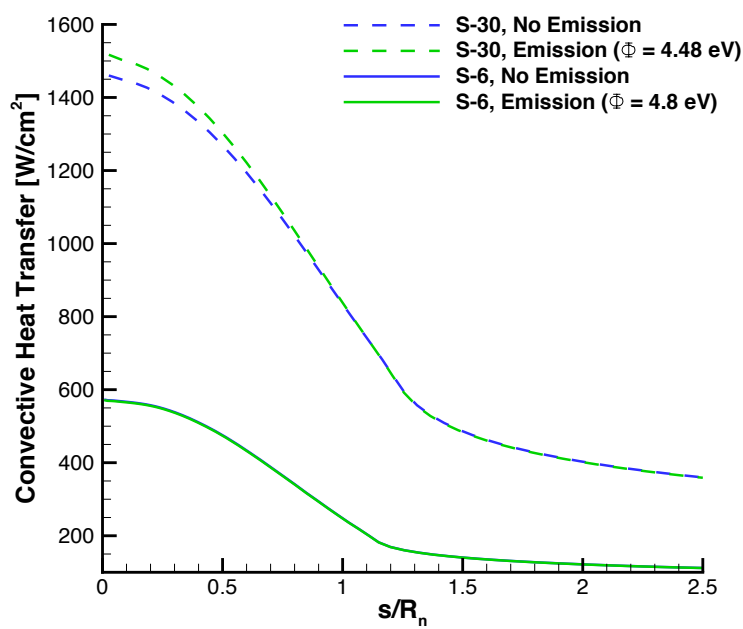

(b) Heat transfer

Figure 5: Surface temperature and heat transfer profiles for the nitrogen Mach 2.75 cases

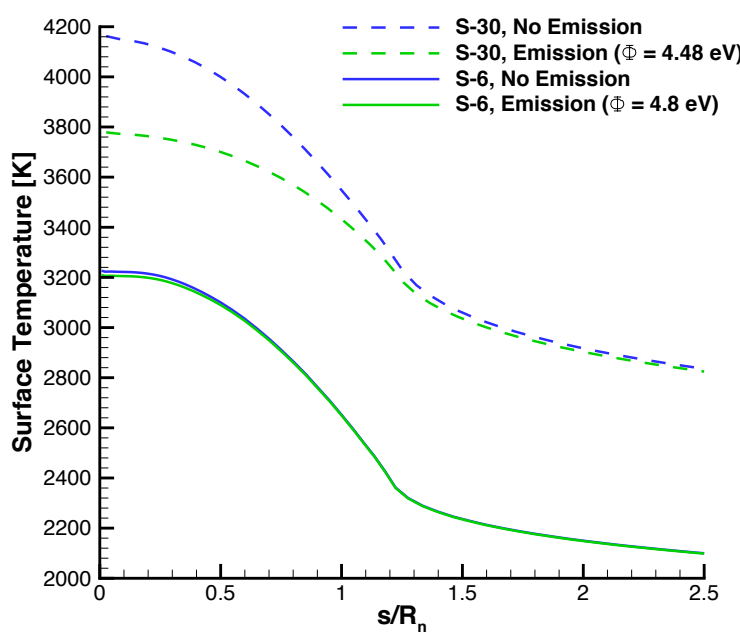

(a) Surface temperature

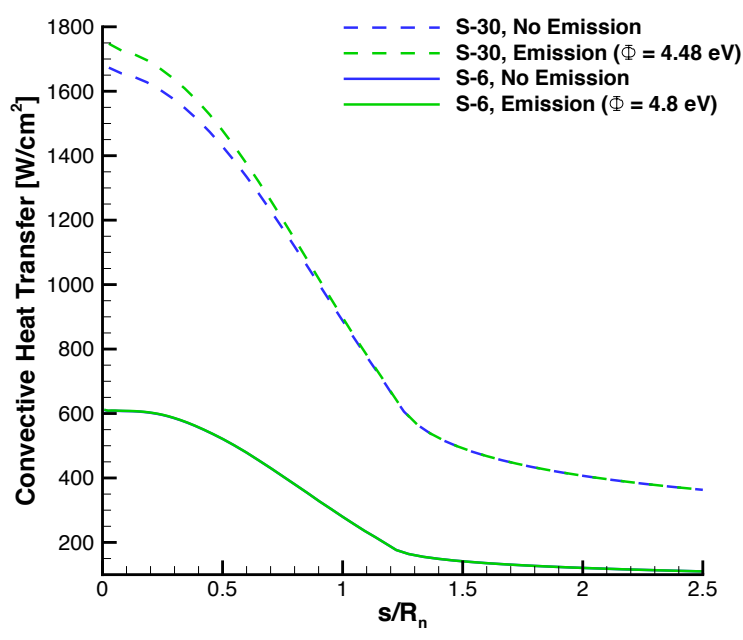

(b) Heat transfer

Figure 6: Surface temperature and heat transfer profiles for the argon Mach 2.75 cases 


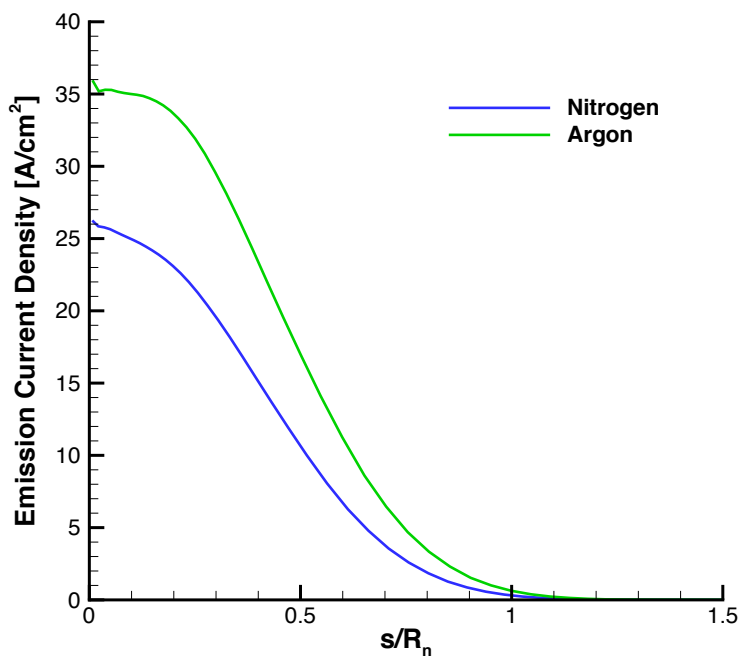

(a) S-6

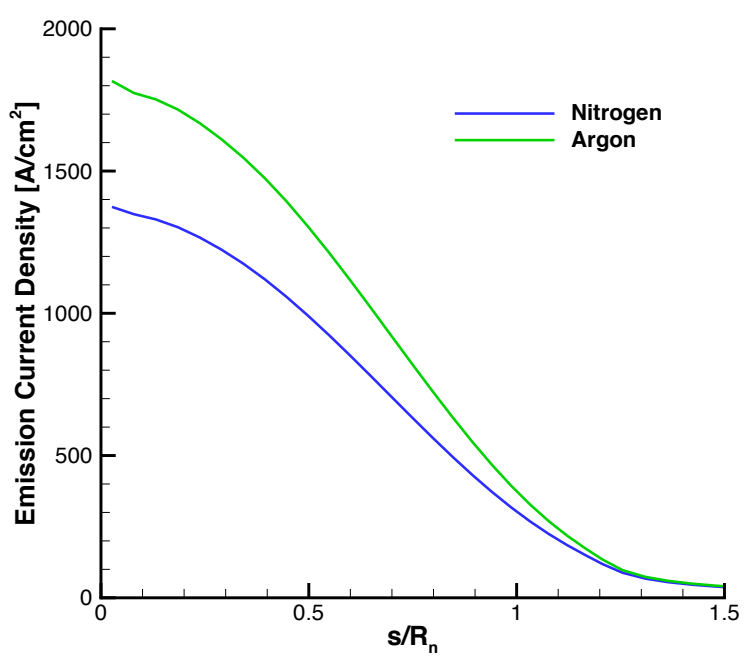

(b) S-30

Figure 7: Surface profiles of emission current density

The experiments cite a short-circuit current from the emitter region and is compared to the computational results in Tables 7 and 8 for the nitrogen cases. For the S-6 emitter current, with graphite as the material, the computational results cover a range from $1.46 \mathrm{~A} / \mathrm{in}^{2}$ for the Mach 2.5 case with a work function of 5.0 $\mathrm{eV}$ to $33.1 \mathrm{~A} / \mathrm{in}^{2}$ for the Mach 3 case with a work function of $4.65 \mathrm{eV}$. The computational values agree reasonably well with the experimental value of $4.0 \mathrm{~A} / \mathrm{in}^{2}$. For the S-30 emitter current, with tungsten as the material, the computational results cover a range from $4.83 \mathrm{~A} / \mathrm{in}^{2}$ for the Mach 2.5 case with a work function of $4.65 \mathrm{eV}$ to $27.3 \mathrm{~A} / \mathrm{in}^{2}$ for the Mach 3 case with a work function of $4.32 \mathrm{eV}$. The computational values are slightly higher than the measured value of $4.5 \mathrm{~A} / \mathrm{in}^{2}$.

Table 7: Graphite S-6 emitter current for nitrogen

\begin{tabular}{c|ccc}
\hline Mach & \multicolumn{3}{|c}{ Work function, $\mathrm{eV}$} \\
Number & 4.65 & 4.8 & 5.0 \\
\hline 2.5 & 5.85 & 3.24 & 1.46 \\
2.75 & 14.8 & 8.49 & 4.01 \\
3.0 & 33.1 & 19.8 & 9.83 \\
\hline Experimental & \multicolumn{3}{|c}{$4.0 \mathrm{~A} / \mathrm{in}^{2}$} \\
\hline
\end{tabular}

Table 8: Tungsten S-30 emitter current for nitrogen

\begin{tabular}{c|ccc}
\hline Mach & \multicolumn{3}{|c}{ Work function, $\mathrm{eV}$} \\
Number & 4.32 & 4.48 & 4.65 \\
\hline 2.5 & 10.6 & 7.29 & 4.83 \\
2.75 & 17.6 & 12.5 & 8.68 \\
3.0 & 27.3 & 19.9 & 14.3 \\
\hline Experimental & \multicolumn{3}{|c}{$4.5 \mathrm{~A} / \mathrm{in}^{2}$} \\
\hline
\end{tabular}

The experimental short-circuit current from the emitter region is compared to the argon computational results in Tables 9 and 10. For the S-6 emitter current, with graphite as the material, the computational 
results cover a range from $3.67 \mathrm{~A} / \mathrm{in}^{2}$ for the Mach 2.5 case with a work function of $5.0 \mathrm{eV}$ to $33.4 \mathrm{~A} / \mathrm{in}^{2}$ for the Mach 3 case with a work function of $4.65 \mathrm{eV}$. The computational results agree well with the experimental value of $21 \mathrm{~A} / \mathrm{in}^{2}$. For the S-30 emitter current, with tungsten as the material, the computational results cover a range from $8.96 \mathrm{~A} / \mathrm{in}^{2}$ for the Mach 2.5 case with a work function of $4.65 \mathrm{eV}$ to $24.9 \mathrm{~A} / \mathrm{in}^{2}$ for the Mach 3 case with a work function of $4.32 \mathrm{eV}$. The computational results agree well with the experimental value of $21 \mathrm{~A} / \mathrm{in}^{2}$.

Table 9: Graphite S-6 emitter current for argon

\begin{tabular}{c|ccc}
\hline Mach & \multicolumn{3}{|c}{ Work function, $\mathrm{eV}$} \\
Number & 4.65 & 4.8 & 5.0 \\
\hline 2.5 & 13.6 & 7.80 & 3.67 \\
2.75 & 21.1 & 12.4 & 5.97 \\
3.0 & 33.4 & 20.1 & 9.98 \\
\hline Experimental & \multicolumn{3}{|c}{$21 \mathrm{~A} / \mathrm{in}^{2}$} \\
\hline
\end{tabular}

Table 10: Tungsten S-30 emitter current for argon

\begin{tabular}{c|ccc}
\hline Mach & \multicolumn{3}{|c}{ Work function, $\mathrm{eV}$} \\
Number & 4.32 & 4.48 & 4.65 \\
\hline 2.5 & 17.5 & 12.7 & 8.96 \\
2.75 & 21.3 & 15.8 & 11.4 \\
3.0 & 24.9 & 18.7 & 13.7 \\
\hline Experimental & \multicolumn{3}{|c}{$21 \mathrm{~A} / \mathrm{in}^{2}$} \\
\hline
\end{tabular}

The computational results are generally higher than the experimental value for the nitrogen cases and lower for the argon cases. The computational results are an overestimation because the the current numerical method does not model recombination of electrons, which would reduce the emitted current. There is also much uncertainty in the measurements in the experiments, including freestream conditions, geometry, and current. The numerical method also assumes the work function of the material is constant whereas it may change as the material degrades under high-temperature exposure.

\section{Conclusions and Future Work}

The goal of the present work was to assess current electron transpiration cooling modeling approaches using a set of experiments performed in the 1960s for a range of freestream conditions, emissive materials, and geometries. The experiments measured the electron current from the emitter region of the geometry and comparisons were made to the modeling approach for nitrogen and argon fluids, graphite and tungsten emissive materials, and two different geometries. The computational results produced a wide range of emitted current due to the uncertainty in the freestream conditions and emissive material, but still agreed well with the experiments. The results also showed that a certain minimum amount of emission is required for the surface to be cooled by electron transpiration cooling.

Although the current modeling capability agreed well with the experiments, further work is needed to improve the modeling capabilities of the numerical method. This includes modeling the recombination of electrons to the surface to better determine the cooling effect of electron emission. Future work will also incorporate better physical models to calculate the effects of the electric field including modeling the forced diffusion of the charged species. This also includes evaluating the effect of electron emission on the electric potential at the surface. 


\section{Acknowledgements}

The authors gratefully acknowledge support for this work from the Lockheed-Martin Corporation. In addition, we thank Dr. Luke Uribarri and Dr. Edward Allen of Lockheed for essential technical oversight. The authors also thank Dr. Erin Farbar and Dr. Kentaro Hara from the University of Michigan for several useful discussions. Finally, the authors thank Dr. Hicham Alkandry of Raytheon for his support and previous work for this study.

\section{References}

${ }^{1}$ Fay, J. A. and Riddell, F. R., "Theory of Stagnation Point Heat Transfer in Dissociated Air," Journal of the Aeronautics Sciences, Vol. 25, No. 2, 1958, pp. 73-85.

${ }^{2}$ Voland, R. T., Huebner, L. D., and McClinton, C. R., "X-43A Hypersonic Vehicle Technology Development," Acta Astronautica, Vol. 57, 2005, pp. 614-622.

${ }^{3}$ Glass, D. E., "Physical Challenges and Limitations Confronting the Use of UHTCs on Hypersonic Vehicles," AIAA Paper 2011-2304, April 2011.

${ }^{4}$ Alkandry, H., Hanquist, K. M., and Boyd, I. D., "Conceptual Analysis of Electron Transpiration Cooling for the Leading Edges of Hypersonic Vehicles," AIAA Paper 2014-2674, June 2014.

${ }^{5}$ Touryan, K. J., "A Hypersonic Plasma Power Generator," AIAA Journal, Vol. 3, No. 4, 1964, pp. 652-659.

${ }^{6}$ LeBlanc, A. R. and Grannemann, W. W., "Thermionic Generator for Re-Entry Vehcicles," Proceedings of the IEEE, Vol. 52, No. 11, 1964, pp. 1302-1310.

${ }^{7}$ Touryan, K. J., "The Hypersonic Plasma Converter: II," Report No. SC-RR-4960, Sandia Laboratories, Albuquerque, New Mexico, 1964.

${ }^{8}$ Martin, A., Scalabrin, L. C., and Boyd, I. D., "High Performance Modeling of Atmospheric Re-Entry Vehicles," Journal of Physics: Conference Series, Vol. 341, No. 1, 2012, Article 012002.

${ }^{9}$ Wilke, C. R., "A Viscosity Equation for Gas Mixtures," Journal of Chemical Physics, Vol. 18, No. 4, 1950, pp. 517-519. 2002.

${ }^{10}$ Vincenti, W. G. and Kruger, C. H., Introduction to Physical Gas Dynamics, Krieger Publishing Company, New York,

${ }^{11}$ Blottner, F. G., Johnson, M., and Ellis, M., "Chemically Reacting Viscous Flow Program for Multi-Component Gas Mixtures," Report No. SC-RR-70-754, Sandia Laboratories, Albuquerque, New Mexico, 1971.

${ }^{12}$ MacCormack, R. W. and Candler, G. V., "The Solution of the Navier-Stokes Equations using Gauss-Seidel Line Relaxation," Computers and Fluids, Vol. 17, No. 1, 1989, pp. 135-150.

${ }^{13}$ Karypis, G. and Kumar, V., METIS: A Software Package for Partitioning Unstructured Graphs, Partitioning Meshes, and Computing Fill-Reducing Ordering of Sparse Matrices, University of Minnesota, Minneapolis, MN, 1998.

${ }^{14}$ Park, C, Nonequilibrium Hypersonic Aerothermodynamics, John Wiley \& Sons, New York, 1990.

${ }^{15}$ Park, C. and Lee, S. H., "Validation of Multi-Temperature Nozzle Flow Code NOZNT," Journal of Thermophysics and Heat Transfer, Vol. 9, No. 1, 1995, pp. 9-16.

${ }^{16}$ George, C., Candler, G., and Pfender, E., "An Implicit Algorithm to Simulate Reaction Chemistry in a Plasma," Journal of Physics D: Applied Physics, Vol. 31, 1998, pp. 2269-2280.

${ }^{17}$ Richardson, O. W., The Emission of Electricity from Hot Bodies, Longmans, Green and Co., London, 1921.

${ }^{18}$ Haynes, W. M., CRC Handbook of Chemistry and Physics, CRC Press, Boca Raton, FL, 2013.

${ }^{19}$ Scott, C. D., "Wall Catalytic Recombination and Boundary Conditions in Nonequilibrium Hypersonic Flows - with Applications," Advances in Hypersonics - Modeling Hypersonic Flows, Vol. 2, 1992, pp. 176-250.

${ }^{20}$ Razier, Y. P., Gas Discharge Physics, Springer-Verlag, Berlin, 1991.

${ }^{21}$ Lieberman, M. A. and Lichtenberg, A. J., Principles of Plasma Discharges and Materials Processing, John Wiley \& Sons, New York, 1994.

${ }^{22}$ Benilov, M. S. and Marotta, A., "A Model of the Cathode Region of Atmospheric Pressure Arcs," Journal of Physics D: Applied Phyiscs, Vol. 28, No. 9, 1995, pp. 1869-1882.

${ }^{23}$ McBride, B. J., and Gordon, S., "Computer Program for Calculation of Complex Chemical Equilibrium Compositions and Applications," NASA Reference Publ. 1311, June 1996.

${ }^{24}$ Moore, C. E., "Atomic Energy Levels as Derived from the Analysis of Optical Spectra, Volume 1," US Bureau of Standards Circular, 1949

${ }^{25}$ Krishnan, K. S. and Jain, S. C., "Thermionic Constants of Graphite," Nature, Vol. 169, No. 4304, 1952, pp. $702-703$.

${ }^{26}$ Reiman, A. L., "Thermionic Emission from Carbon," Proceedings of the Physical Society, Vol. 50, No. 4, 1938, pp. 496-500.

${ }^{27}$ Nichols, M. H., "The Thermionic Constants of Tungsten as a Functions of Crystallographic Direction," Physical Review Letters, Vol. 57, No. 4, 1940, pp. 297-306.

${ }^{28}$ Nottingham, W. B., "Remarks on Energy Losses Attending Thermionic Emission of Electrons from Metals," Physical Review Letters, Vol. 59, No. 11, 1941, pp. 906-907. 